\title{
Neoadjuvant treatment is always justified for small PDAC, especially for clinical T1? - Debate from the position of Pros
}

\author{
$\underline{\text { Jin-Seok HEO* }}^{*}$ \\ Sungkyunkwan University, Korea
}

Lecture: Surgical resection offers the best chance for long-term survival, and upfront surgery is the most universally accepted approach for potentially resectable PDAC. But the result is dismal, the median survival of patients undergoing curative pancreatectomy alone is 18 to 20 months, with a 5 -year survival rate of $10 \%$.

The administration of adjuvant chemotherapy and possibly chemoradiation leads to an improvement in OS relative to pancreatectomy alone. Adjuvant therapy following pancreatectomy therefore currently represents standard of care for patients with resectable PDAC. But, Administration of planned adjuvant chemotherapy may be limited by postoperative complications and early recurrence. Unfortunately, as few as $50 \%$ of patients who undergo pancreatectomy nationwide actually receive postoperative therapy. In an effort to overcome these barriers, early delivery of chemotherapy was evaluated as an alternative treatment sequence strategy. So the neoadjuvant therapies have been proposed.

Two major hypothetical risks have been pointed out for neoadjuvant chemotherapy (NAC). One is a possible increase in perioperative morbidity and mortality. Second is the possibility that disease may progress and become unresectable during the course of NAC. A nationwide survey suggested that neoadjuvant treatment might not worsen perioperative outcomes or might increase the chance for curative surgery.

NAC offers several theoretical advantages over upfront surgery, including early delivery of systemic therapy for almost all patients intended for treatment, high tolerance of multi-agent regimens by patients and a higher negative-margin resection rate, leading to improve OS. A review of select trials for patients with localized PDAC has suggested increased OS, supporting the benefits.

Of course to date, there are no prospective data providing the superiority of neoadjuvant strategy over upfront surgery for resectable PDAC. So neoadjuvant treatment is not always justified for small PDAC, up to now. Nevertheless, neoadjuvant treatment can be a practical treatment strategy, particularly for patients at high biological or perioperative risk.

\section{Reference}

1. Cheng TY, Sheth K, White RR, Ueno T, Hung CF, Clary BM, et al. Effect of neoadjuvant chemoradiation on operative mortality and morbidity for pancreaticoduodenectomy. Ann Surg Oncol 2006;13:66-74.

2. Gillen S, Schuster T, Meyer Zum Büschenfelde C, Friess H, Kleeff J. Preoperative/neoadjuvant therapy in pancreatic cancer: a systematic review and meta-analysis of response and resection percentages. PLoS Med 2010;7:e1000267.

3. Glant JA, Waters JA, House MG, Zyromski NJ, Nakeeb A, Pitt HA, et al. Does the interval from imaging to operation affect the rate of unanticipated metastasis encountered during operation for pancreatic adenocarcinoma? Surgery 2011;150:607-616.

4. Tzeng CW, Tran Cao HS, Lee JE, Pisters PW, Varadhachary GR, Wolff RA, et al. Treatment sequencing for resectable pancreatic cancer: influence of early metastases and surgical complications on multimodality therapy completion and survival. J Gastrointest Surg 2014;18:16-24; discussion 24-5.

5. Winner M, Goff SL, Chabot JA. Neoadjuvant therapy for non-metastatic pancreatic ductal adenocarcinoma. Semin Oncol 
2015;42:86-97.

6. Tsai S, Evans DB. Therapeutic advances in localized pancreatic cancer. JAMA Surg 2016;151:862-868.

7. de Geus SW, Eskander MF, Bliss LA, Kasumova GG, Ng SC, Callery MP, et al. Neoadjuvant therapy versus upfront surgery for resected pancreatic adenocarcinoma: a nationwide propensity score matched analysis. Surgery 2017;161:592-601.

8. Mokdad AA, Minter RM, Zhu H, Augustine MM, Porembka MR, Wang SC, et al. Neoadjuvant therapy followed by resection versus upfront resection for resectable pancreatic cancer: a propensity score matched analysis. J Clin Oncol 2017;35:515-522.

9. Motoi F, Kosuge T, Ueno H, Yamaue H, Satoi S, Sho M, et al.; Study Group of Preoperative Therapy for Pancreatic Cancer (Prep) and Japanese Study Group of Adjuvant Therapy for Pancreatic Cancer (JSAP). Randomized phase II/III trial of neoadjuvant chemotherapy with gemcitabine and S-1 versus upfront surgery for resectable pancreatic cancer (Prep-02/JSAP05). Jpn J Clin Oncol 2019;49:190-194.

10. Greco SH, August DA, Shah MM, Chen C, Moore DF, Masanam M, et al. Neoadjuvant therapy is associated with lower margin positivity rates after pancreaticoduodenectomy in T1 and T2 pancreatic head cancers: an analysis of the National Cancer Database. Surg Open Sci 2020;3:22-28. 